\title{
Les diatomées pennales de la lagune de Fresco (Côte d'Ivoire)
}

\author{
Estelle Sévérine KONAN ${ }^{1 *}$, N'Guessan Maryse $\mathrm{AKA}^{1}$ et Marie Paulette $\mathrm{ADON}^{2}$ \\ ${ }^{1}$ Centre de Recherches Océanologiques, 29, Rue des Pêcheurs Treichville BPV 18 Abidjan, Côte d'Ivoire. \\ ${ }^{2}$ Département Biodiversité et Gestion durable des Ecosystèmes, UFR-Environnement, Université Jean \\ Lorougnon Guédé, BP 150 Daloa, Côte d'Ivoire. \\ *Auteur correspondant ; E-mail : estydekonan@yahoo.fr
}

\section{RÉSUMÉ}

Ce travail vise à faire l'étude taxonomique des diatomées pennales récoltées dans la lagune de Fresco en Côte d'Ivoire. Le manque d'information sur ce groupe d'algues, justifie la présente étude. Des échantillonnages mensuels ont été réalisés de Mars 2007 à Décembre 2008 sur cinq stations. Le phytoplancton a été récolté à l'aide d'un filet à plancton de $20 \mu \mathrm{m}$ de vide de maille et les échantillons ont été fixés à l'aide $\mathrm{du}$ formol de commerce à la concentration finale de $5 \%$. Un microscope ordinaire BX40 muni d'un micromètre objet, a été utilisé pour les observations des différents taxons. Les identifications ont été faites à l'aide de divers ouvrages de différents auteurs. A l'issu des différentes manipulations, 30 taxons répartis en 2 classes, 5 ordres, 8 familles, et 16 genres ont été identifiés. La communauté algale de la lagune de Fresco est dominée par les espèces marines (14 taxons). Les stations 4 et 5 présentent les richesses floristiques les plus élevées, avec 21 taxons pour chacune des stations. Meridion circulare, Pinnularia legumen, Navicula humerosa, Gyrosigma diminutum, Campylodiscus eximus, Surirella ovata, Surirella robusta, sont signalées pour la première fois en Côte d'Ivoire.

(C) 2019 International Formulae Group. All rights reserved.

Mots clés: Diatomées pennales, Lagune de Fresco, Phytoplancton, Côte d'Ivoire.

\section{Pennate diatoms of Fresco Lagoon (Côte d'Ivoire)}

\begin{abstract}
This paper represents the morphological identifications of pennate diatoms from Fresco Lagoon. Absence of information about pennate diatoms in Fresco Lagoon, justify this study. An investigation was carried out monthly from March 2007 to December 2008. Five stations were chosen for sampling. Phytoplankton was collected using a $20 \mu \mathrm{m}$ plankton net coupled with a collector. The samples were preserved with $5 \%$ buffered formalin. Some plankton samples were subjected to acid wash before detailed morphological observations. Phytoplankton samples were examined in the laboratory using an Olympus BX40 microscope equipped with a calibrated micrometer for taxa observations. Identification was made with reference to different authors. Observation and identification revealed 30 taxa belonging 2 classes, 5 orders, 8 families and 16 genera. The algal community was dominated by marine species (14 taxa). Stations 4 and 5 recorded the highest number of taxa (21 taxa). Meridion circulare, Pinnularia legumen, Navicula humerosa, Gyrosigma diminutum, Campylodiscus eximus, Surirella ovata, Surirella robusta, are reported for the first time for Côte d'Ivoire.

(C) 2019 International Formulae Group. All rights reserved.
\end{abstract}

Keywords: Pennate diatoms, Fresco Lagoon, Phytoplankton, Côte d'Ivoire. 


\section{INTRODUCTION}

Les Diatomées sont des organismes microscopiques, eucaryotes, photosynthétiques qui se développent dans les milieux aquatiques. Elles constituent un grand groupe écologique et peuvent se présenter en cellules isolées ou regroupées en colonies. Elles se caractérisent par une paroi rigide faite de silice hydratée insérée dans une matrice organique : le frustule divisé en deux valves (l'hypothèque et l'épithèque) emboitées et de tailles différentes (Hans et Bayer, 2002). Les diatomées constituant une part importante du phytoplancton, contribuent largement à la fixation du dioxyde de carbone atmosphérique, et donc au cycle du carbone, et au cycle du silicium (Smol et Stoermer, 2010). Elles sont responsables d'environ $20-25 \%$ de la production global de l'oxygène et représentent $40 \%$ de l'ensemble des espèces phytoplanctoniques. Elles constituent, le groupe le plus important en terme de diversité, d'abondance et de productivité dans les milieux marins et continentaux (Round et al., 1990). Elles jouent un rôle très important dans le réseau trophique existant dans l'eau. En effet, elles servent d'alimentation de base à certains microorganismes d'ordre supérieur (Taylor et al., 2007). De par leur sensibilité aux nutriments, due aux variations de la composition chimique des eaux, (Zongo et al., 2011) et à la matière organique, les diatomées sont d'excellents indicateurs de la qualité de l'eau et du niveau d'eutrophisation des cours d'eau (Chahboune et al., 2012 ; Campeau et al., 2014). Elles sont très utilisées pour les reconstitutions paléo-environnementales et paléo océanographiques (Saint Martin et al, 2004).

En Côte d'Ivoire, les études algales en milieu saumâtre qui ont été menées concernent principalement 3 milieux lagunaires qui sont les lagunes Ébrié (PlanteCuny, 1977; Maurer, 1978; Dufour et al., 1979 ; Arfi et al., 1981; Carpentier, 1982 ; Iltis, 1984 ; Tanoh, 2006 ; N'Gouran, 2006 et Seu-Anoï, 2012) , Grand-Lahou (Komoé, 2010 et Seu-Anoï, 2012) et Aby (Adou, 1999 et Seu-Anoï, 2012).
Bien que le suivi des populations phytoplanctoniques ait débuté à partir de 1964 par le Centre de Recherches Océanologiques (Côte d'Ivoire), sur la zone côtière, semicôtière, et le large (Reyssac, 1966), aucune étude relative aux diatomées pennales n'a été faite sur la lagune de Fresco. L'importance écologique de ce groupe et l'absence d'information sur la systématique de ces diatomées justifie l'intérêt de ce travail dont l'objectif principal est de déterminer la composition taxonomique des diatomées pennales de la lagune de Fresco.

\section{MATÉRIEL ET MÉTHODES Description des sites}

La lagune de Fresco, située entre les longitudes $5^{\circ} 32^{\prime}$ et $5^{\circ} 38^{\prime}$ Ouest et les latitudes $5^{\circ} 40^{\prime}$ et $5^{\circ} 70^{\prime}$ Nord, a un plan d'eau lagunaire qui s'étire d'Est en Ouest, sur une longueur d'environ $6 \mathrm{~km}$, une largeur de 2 à $4 \mathrm{~km}$, avec une profondeur moyenne de $4 \mathrm{~m}$. Elle couvre une superficie de $17 \mathrm{~km}^{2}$ et reçoit les eaux des quatre rivières côtières dont deux principales : le Niouniourou $(140 \mathrm{~km})$, le Bolo $(84 \mathrm{~km})$ et deux moins importantes : le Gnou $(12 \mathrm{~km})$ et le Guitako $(5 \mathrm{~km})$. La lagune de Fresco communique avec l'Océan Atlantique par la passe non permanente de Fresco. Le volume d'eau océanique entrant varie avec le niveau relatif des plans d'eau lagunaire et océanique. Il est maximal au cours des périodes d'étiage des rivières Bolo et Niouniourou et minimal au cours des périodes de crues (Abé et al., 1993).

Les prélèvements ont été effectués sur cinq stations (Figure 1). Le choix de ces stations est basé sur la situation géographique, les activités anthropiques et l'entrée des cours d'eau.

\section{Méthodes de collecte des Diatomées}

Les récoltes ont été faites à l'aide d'un filet à plancton de $20 \mu \mathrm{m}$ de vide de maille. L'eau de surface a été transvasée dans le filet par un seau d'une contenance de 10 litres. Les algues ont été recueillies par filtration au filet dans des piluliers de $60 \mathrm{~mL}$ et ont été fixées au formol de commerce (neutralisé par du 
borate de soude) à la concentration finale de $5 \%$. Cette méthode permet de concentrer le plus d'organismes possibles.

\section{Méthodes d'observation et d'identification des diatomées}

Les diatomées ont fait l'objet de préparations spécifiques pour quelques échantillons dans le but de mieux faire apparaître les détails de l'ornementation (stries, pores) de la coque siliceuse ou frustule (Rumeau et Coste, 1988). De l'acide nitrique a été utilisé afin d'éliminer tous les composants organiques (Leclercq et Maquet, 1987). Les diatomées sont par la suite observées, photographiées et mesurées, au microscope de type Olympus BX40.

L'identification des Diatomées a nécessité l'utilisation des travaux de : Peragallo (1908), Bourrelly (1961, 1975, 1981), Amossé (1970), Compère (1975b, 1991), Couté et Iltis (1985), Krammer et Lange-Bertalot (1991), Round et al. (1990), Komoé (2010). La classification suivie est celle de Round et al. (1990).

Dans la partie résultat les taxons précédés d'un astérisque $(*)$ sont signalés pour la première fois en Côte d'Ivoire. Au niveau de chaque planche, le trait à coté de chaque image représente l'échelle qui est de $10 \mu \mathrm{m}$.

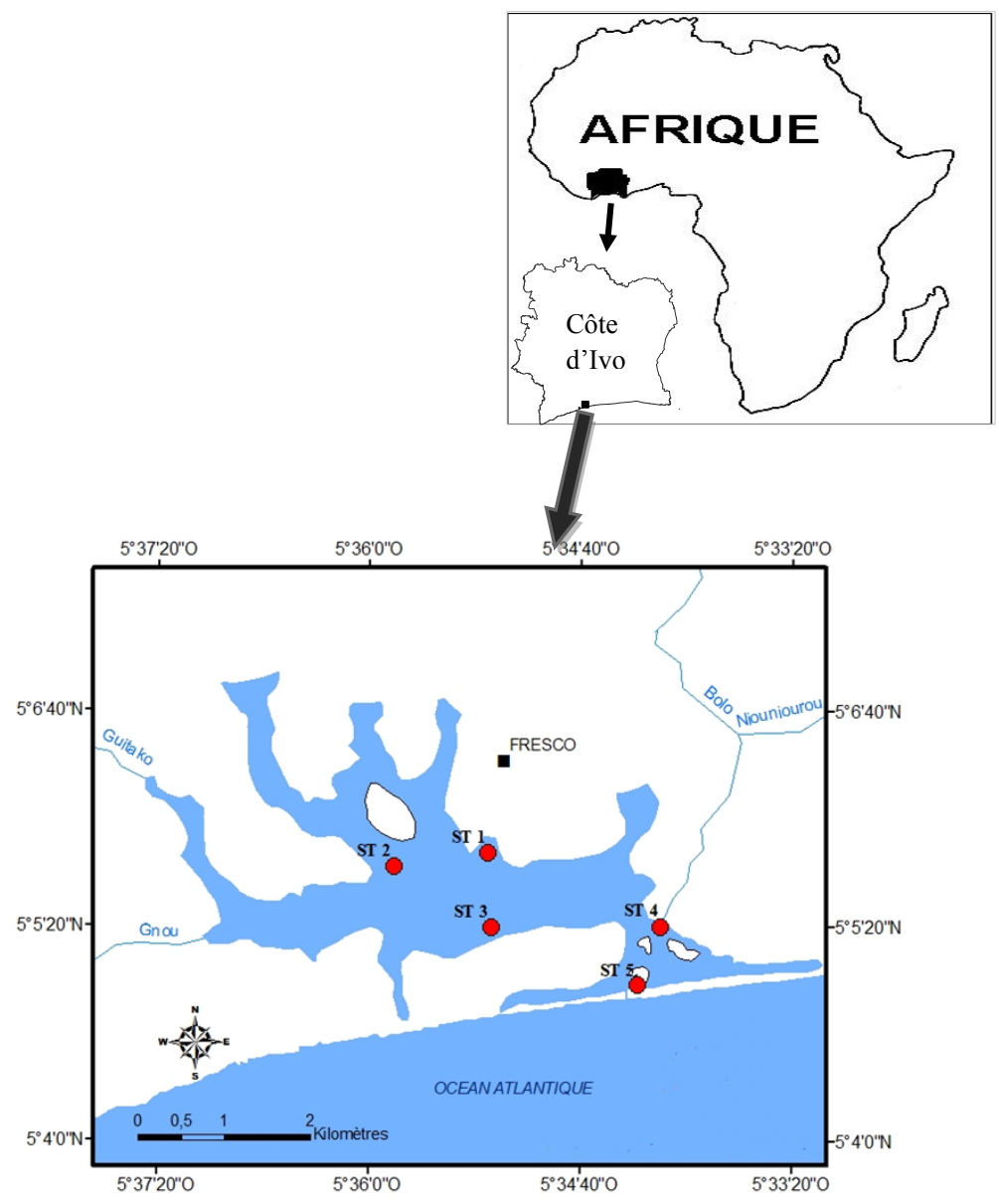

Figure 1: Localisation des stations d'échantillonnage. 


\section{RÉSULTATS}

Classe: Fragilariophyceae Round

Ordre: Fragilariales Silva

Famille: Fragilariaceae Greville

Asterionellopsis glacialis (Castracane)

Round

Les cellules rectilignes, sont groupées en colonies étoilées (Figure 2). Les valves hétéropolaires, très étroites sur presque toute leur longueur, montrent un élargissement très prononcé à la base. Elles mesurent 58 x 5-7 $\mu \mathrm{m}$. C'est une espèce marine, cosmopolite, prélevée aux stations $1,2,3,4$ et 5 .

\section{Fragilaria crotonensis Kitton}

Ce sont des cellules coloniales réunies entre elles, avec des frustules étroits et rectangulaires en vue connective, linéaires à linéaires lancéolés (Figure 3). Elles mesurent 98-120 x 3,8-4 $\mu \mathrm{m}$. C'est une espèce d'eau douce, cosmopolite, récoltée aux stations 3 et 4.

\section{Fragilaria dorsiventralis (O. Müller) Lange- Bertalot}

Les cellules isopolaires sont réunies par leurs valves en colonies et sont fusiformes en vue valvaire (Figure 4). Elles mesurent 6264 x 10-12 $\mu \mathrm{m}$. C'est une espèce récoltée à la station 5 .

\section{Ulnaria ulna (Nitzsch) Compère}

Ce sont des espèces dont les frustules sont linéaires en vue valvaire (Figure 5). Elles mesurent 92 × $9 \mu \mathrm{m}$. C'est une espèce d'eau douce, cosmopolite, échantillonnée aux stations 1, 2, 3, 4 et 5 .

\section{*Meridion circulare (Greville) Agardh}

Les cellules hétéropolaires, adhèrent par leurs valves et forment des colonies en éventail ou parfois même en cercle (Figure 6). Elles mesurent 44 x $8 \mu \mathrm{m}$. C'est une espèce d'eau douce, cosmopolite, prélevée aux stations 4 et 5 .

\section{Ordre: Thalassionematales Round}

Famille: Thalassionemataceae Round

\section{Thalassionema Frauenfeldianum Grunow}

Les cellules sont rectilignes, parfois faiblement arquées et rarement libres. Elles sont réunies par leur apex pour former une étoile (Figure 7). Elles mesurent 66-78 x 3-3,4 $\mu \mathrm{m}$. C'est une espèce marine, cosmopolite, récoltée aux stations $1,2,3,4$ et 5 .

\section{Thalassionema nitzschioides (Grunow) Hustedt}

Les cellules, coloniales, sont réunies par leurs extrémités pour former des colonies en zig-zag et /ou étoilées (Figure 8). Elles mesurent $50 \times 4 \mu \mathrm{m}$. C'est une espèce marine, cosmopolite, récoltée aux stations $1,2,3,4$ et 5.

\section{Classe: Bacillariophyceae Mann \\ Ordre: Naviculales Bessey \\ Famille: Pinnulariaceae Mann}

*Pinnularia legumen Ehrenberg

Les valves sont très allongées, renflées au centre et aux extrémités. Les stries sont interrompues au centre et le raphé est droit (Figures 9a-9b). Cette espèce mesure 106 x 14 $\mu \mathrm{m}$. C'est une espèce d'eau douce, subcosmopolite, récoltée aux stations 4 et 5 .

\section{Pinnularia maior (Kützing) Rabenhorst}

Les valves sont de grande taille, légèrement renflées au centre, et arrondies aux extrémités (Figure 10). Le raphé est simple, parfois sinueux. Ces cellules mesurent 152198 x 22-36 $\mu \mathrm{m}$. C'est une espèce dulçaquicole, subcosmopolite, récoltée à la station 5.

\section{Pinnularia viridis (Nitzsch) Ehrenberg}

Les valves sont linéaires, arrondies aux pôles (Figure 11), avec une aire axiale assez large. Le raphé est large et droit et compte 7 à 9 stries en $10 \mu \mathrm{m}$. Cette espèce mesure $96 \mathrm{x}$ $20 \mu \mathrm{m}$. C'est une espèce dulçaquicole, cosmopolite récoltée à la station 4 .

\section{Famille : Diploneidaceae Mann Diploneis oblongella (Nägeli) Cleve-Euler}

Les cellules sont solitaires, avec des valves ellipsoïdales (Figure 12). Les stries transapicales sont séparées par une seule rangée de points. On dénombre 11 à 13 stries en $10 \mu \mathrm{m}$. Elles mesurent 42-56 x 18-20 $\mu \mathrm{m}$. 
C'est une espèce à la fois marine et saumâtre, cosmopolite, récoltée à la station 4 .

\section{Diploneis smithii (Brébisson in W. Smith) Cleve}

Ce sont des cellules solitaires, à valves elliptiques et aux côtés convexes, de 44 x 24 $\mu \mathrm{m}$. Les côtes transapicales sont séparées par une seule rangée de points. On dénombre 1317 stries en $10 \mu \mathrm{m}$ (Figure 13). C'est une espèce à la fois saumâtre et marine, cosmopolite, récoltée aux stations 4 et 5 .

\section{Famille: Naviculaceae Kützing Amphiprora alata Kützing}

On dénombre 9 stries en $10 \mu \mathrm{m}$ et la cellule mesure 87 x $38 \mu \mathrm{m}$ (Figure 14). Cette espèce d'eau saumâtre et cosmopolite a été récoltée aux stations 1, 2, 3 et 4.

\section{Amphiprora paludosa Smith}

La vue connective présentant un grand nombre de ceintures secondaires, a un contour en forme de 8 dû à une carène saillante. On dénombre 20 stries en $10 \mu \mathrm{m}$. Cette espèce mesure 62 × $50 \mu \mathrm{m}$ (Figure 15). C'est une espèce saumâtre, cosmopolite, récoltée aux stations 1,2, 3 et 4.

\section{Donkinia sp.}

Ce sont des espèces à valves presque rectilignes, et possédant un raphé sigmoïde. Elles mesurent $104 \times 22 \mu \mathrm{m}$ (Figure 16). Ce taxon a été rencontré à la station 1 .

\section{*Navicula humerosa Brébisson}

Les valves sont larges et linéaires, fortement rétrécies et arrondies aux extrémités. Elles mesurent 58-62 x 24-27 $\mu \mathrm{m}$ et on compte 9 à 10 stries en $10 \mu \mathrm{m}$. Les stries sont transapicales et forment des rangées de points le long du raphé (Figure 17). C'est une espèce d'eau marine, subcosmopolite, récoltée à la station 4 .

\section{Navicula sp.}

$\mathrm{Ce}$ sont des cellules solitaires, ou réunies en de longues chaînes, avec comme dimensions 28-40 x 10-13 $\mu \mathrm{m}$. Les valves sont lancéolées et leurs extrémités arrondies
(Figures 18a-18b). Ce taxon a été récolté aux stations 1, 2, 3 et 4 .

\section{Trachyneis antillarum Cleve}

Ce sont des espèces dont les valves sont linéaires et lancéolées et à apex obtus. Le raphé est excentrique et la zone centrale arrondie (Figures 19a-19b). Les dimensions sont de $80 \times 23 \mu \mathrm{m}$. C'est une espèce marine, subcosmopolite, rencontrée à la station 5 .

\section{Famille: Pleurosigmataceae}

Mereschkowsky

\section{Gyrosigma balticum Ehrenberg}

C'est une espèce à valves presque rectilignes dont les extrémités sont infléchies et le raphé excentré (Figure 20). Elles mesurent $112 \times 8 \mu \mathrm{m}$. C'est une espèce marine, cosmopolite, récoltée aux stations 1 , 2, 3, 4 et 5 .

\section{*Gyrosigma diminutum (Grunow in Cleve \& Grunow) Cleve}

C'est une espèce à valves également presque rectilignes, dont les extrémités sont toutes aussi infléchies (Figure 21). Elle mesure $72 \times 10 \mu \mathrm{m}$. C'est une espèce d'eau saumâtre, récoltée à la station 5 .

\section{Gyrosigma scalproides Cleve}

Ces cellules possèdent des frustules presque droits et sont peu sigmoïdes (Figure 22). Elles mesurent $68 \times 12 \mu \mathrm{m}$. C'est une espèce saumâtre, cosmopolite, récoltée à la station 5 .

\section{Ordre: Bacillariales Hendey}

Famille: Bacillariaceae Ehrenberg Nitzschia sigma (Kützing) W. Smith

C'est une espèce à valves sigmoïdes en vue valvaire et connective (Figure 23). Elles mesurent 198-226 x 6-10 $\mu \mathrm{m}$. C'est une espèce marine, cosmopolite, récoltée aux stations 1,4 et 5 .

\section{Ordre: Surirellales Mann}

Famille: Surirellaceae Kützing

\section{Campylodiscus clypeus Ehrenberg}

Les cellules solitaires, possèdent des valves subcirculaires (Figure 24). La surface 
valvaire présente des côtes en disposition radiale, interrompues au milieu par un pseudoraphé. Elles mesurent 60 x $49 \mu \mathrm{m}$. C'est une espèce saumâtre, marine, cosmopolite, rencontrée à la station 4 .

\section{Campylodiscus echeneis Ehrenberg}

Les cellules solitaires, possèdent des valves subcirculaires. Les valves présentent des côtes granulées, en disposition radiale autour d'une aire centrale elliptique (Figure 25). Elles mesurent $56 \times 47 \mu \mathrm{m}$. C'est une espèce à la fois saumâtre et marine, cosmopolite, récoltée à la station 5 .

\section{*Campylodiscus eximus Gregory}

Les cellules sont solitaires et possèdent des valves subcirculaires. On note la présence de côtes en disposition radiale autour d'une aire centrale subconique (Figure 26). Elles mesurent $60 \times 52 \mu \mathrm{m}$. C'est une espèce saumâtre, marine, subcosmopolite échantillonnée à la station 5 .

\section{Petrodictyon gemma (Ehrenberg) Mann}

Les cellules sont le plus souvent elliptiques ou ovoïdes sur la face valvaire (Figure 27). Les stries sont peu accentuées et sont au nombre de 23 à 29 en $100 \mu \mathrm{m}$. Elles mesurent 105 x $40 \mu \mathrm{m}$. C'est une espèce marine, cosmopolite récoltée aux stations 1,2 , 3,4 et 5 .

\section{Surirella fastuosa (Ehrenberg) Kützing}

Les cellules sont solitaires, ovoïdes et hétéropolaires. Elles possèdent des côtes bien marquées au niveau des marges et effilées dans la zone centrale qui est largement lancéolée. Les stries sont plus évidentes au niveau des marges et vers le centre (Figure 28). Le nombre de côtes varie de 11 à 17 en $100 \mu \mathrm{m}$. Elles mesurent $82 \times 35 \mu \mathrm{m}$. C'est une espèce marine, cosmopolite, récoltée à toutes les stations.

\section{*Surirella ovata Kützing}

$\mathrm{Ce}$ sont des cellules à valves hétéropolaires plus ou moins ovoïdes (Figure 29). Elles mesurent $66 \times 22 \mu \mathrm{m}$. C'est une espèce marine, subcosmopolite, rencontrée à la station 5.

\section{*Surirella robusta Ehrenberg}

Elle mesure 82-130 x 48-64 $\mu \mathrm{m}$ (Figures 30a-30b). C'est une espèce marine et dulçaquicole, subcosmopolite rencontrée aux stations 4 et 5 .

\section{Surirella splendida Kützing}

Les cellules sont le plus souvent elliptiques ou ovoïdes, hétéropolaires sur la face valvaire (Figure 31). Elles mesurent 85 150 x 52-60 $\mu \mathrm{m}$. Les stries sont au nombre de 14 à 21 en $100 \mu \mathrm{m}$ et sont peu accentuées. C'est une espèce marine, cosmopolite récoltée aux stations $1,2,3,4,5$.

\section{Composition floristique}

La communauté des diatomées pennales de la lagune de Fresco comprend au total 30 taxons répartis en 16 genres, 8 familles, 5 ordres et 2 classes. Le genre Surirella avec 4 taxons est le mieux représenté, suivi des genres Campilodiscus, Gyrosigma, Pinnularia avec 3 taxons. Les genres Navicula, Amphiprora, Fragilaria, Diploneis et Thalassionema sont representés chacun par 2 taxons. Sept genres sont représentés par seulement un taxon. Il s'agit des genres Asterionellopsis, Ulnaria, Meridion, Donkinia, Trachyneis, Nitzschia et Petrodictyon. Les stations 1,2 et 3 , ont enregistré respectivement 13, 11 et 12 taxons. Quant aux stations 4 et 5, elles ont enregistré chacune 21 taxons. Quatorze taxons sont d'origine marine, 9 sont saumâtres et 6 d'eaux douces. Dix-neuf sont cosmopolites et 7 subcosmopolites. 

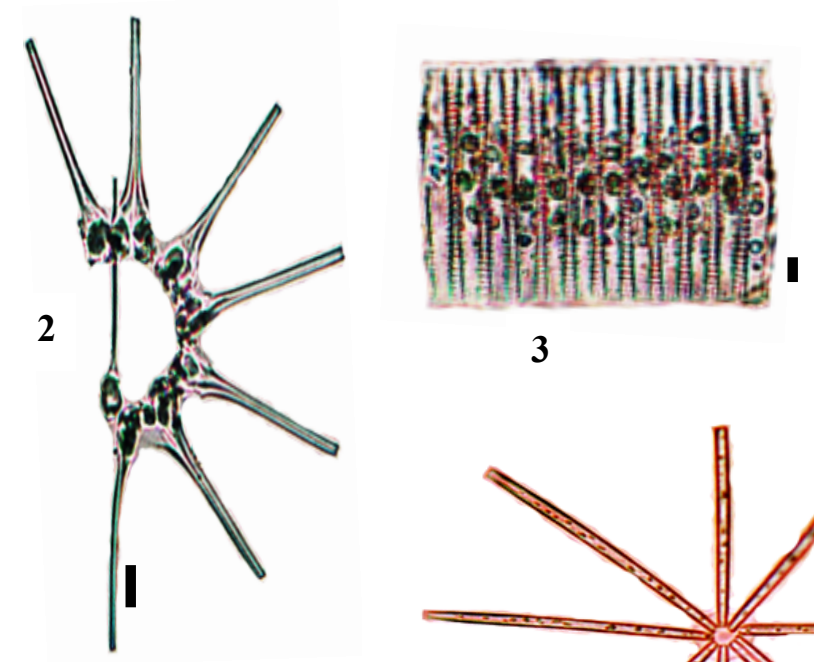

3
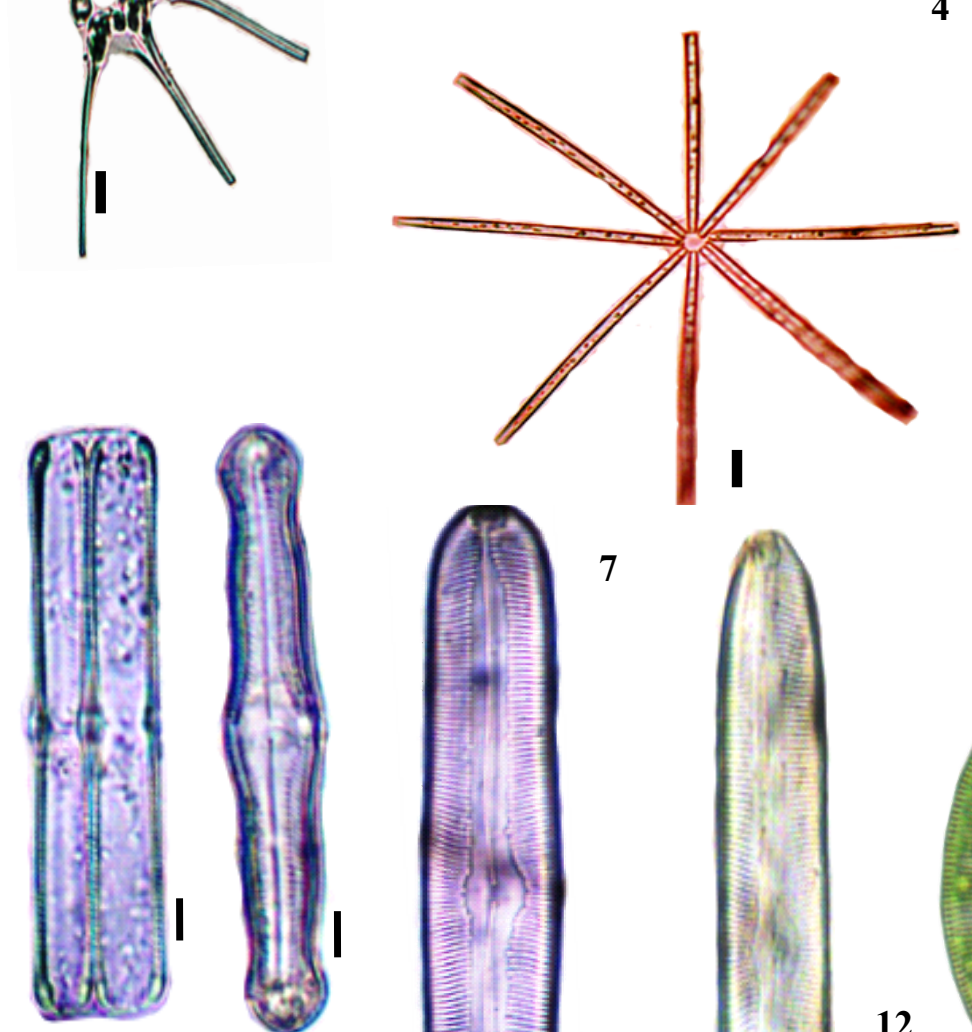

9 a

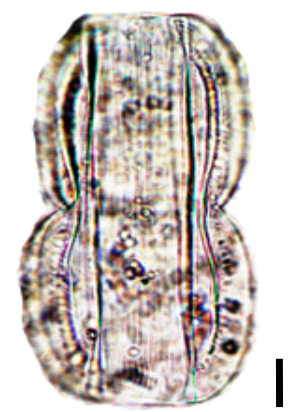

10

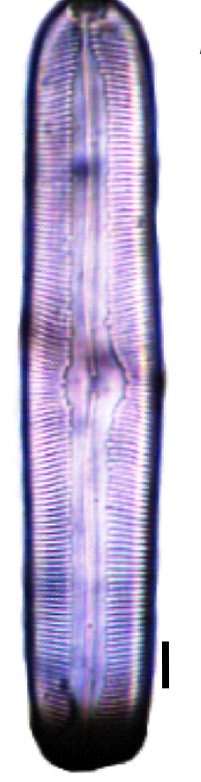

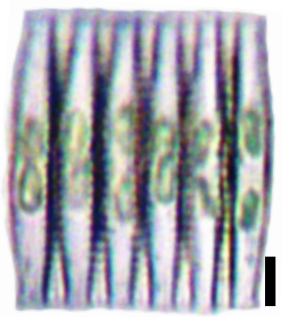

4

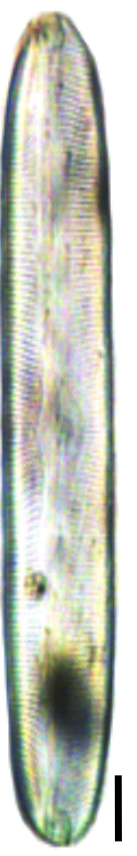

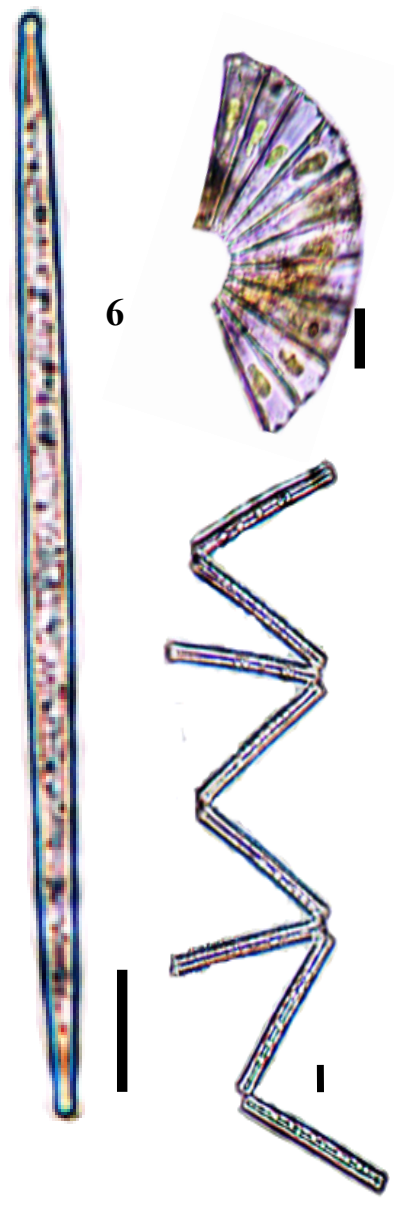

8

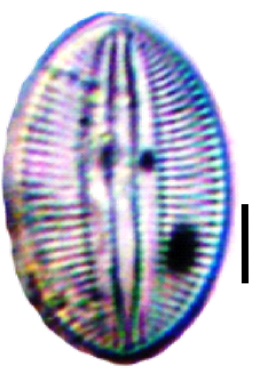

12

11
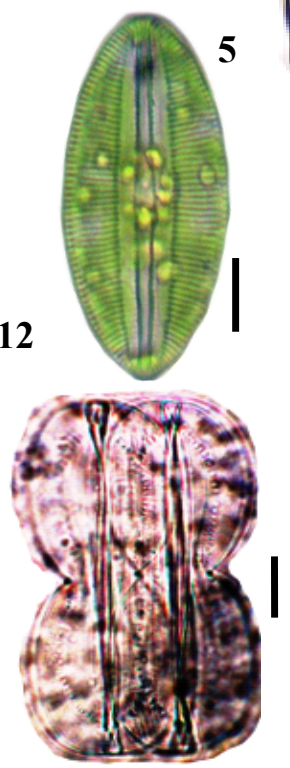

15

Figures 2-15 : 2- Asterionellopsis glacialis (Castracane) Round, 3- Fragilaria crotonensis Kitton, 4- Fragilaria dorsiventralis (O. Müller) Lange-Bertalot, 5- Ulnaria ulna (Nitzsch) Compère, 6- Meridion circulare (Greville) Agardh, 7- Thalassionema frauenfeldianum Grunow, 8-Thalassionema nitzschioides (Grunow) Hustedt, 9a, 9b-Pinnularia legumen Ehrenberg, 10Pinnularia maior (Kützing) Rabenhorst, 11- Pinnularia viridis (Nitzsch) Ehrenberg 12-Diploneis oblongella (Nägeli) CleveEuler, 13-Diploneis smithii (Brébisson in W. Smith) Cleve, 14- Amphiprora alata Kützing, 15-Amphiprora paludosa Smith. 


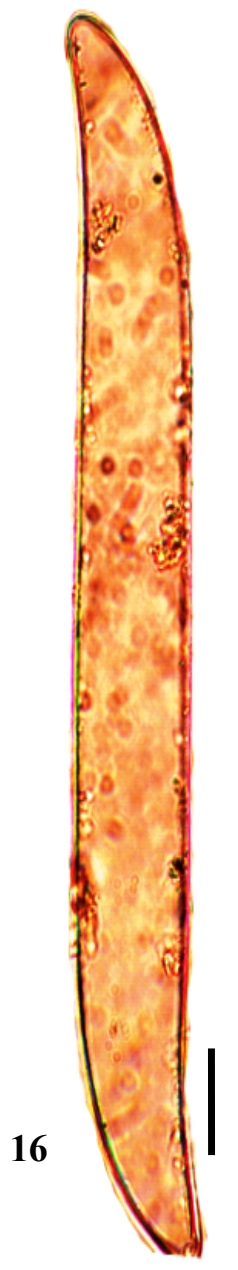

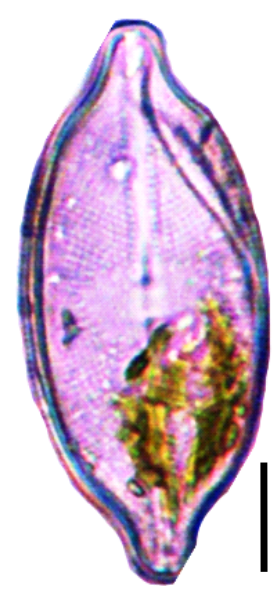

17

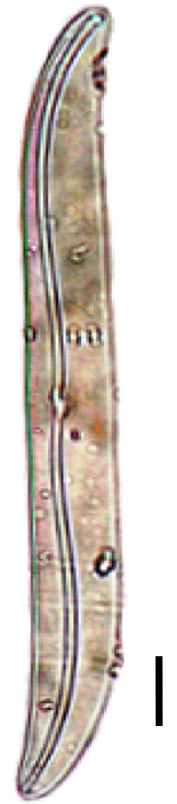

20
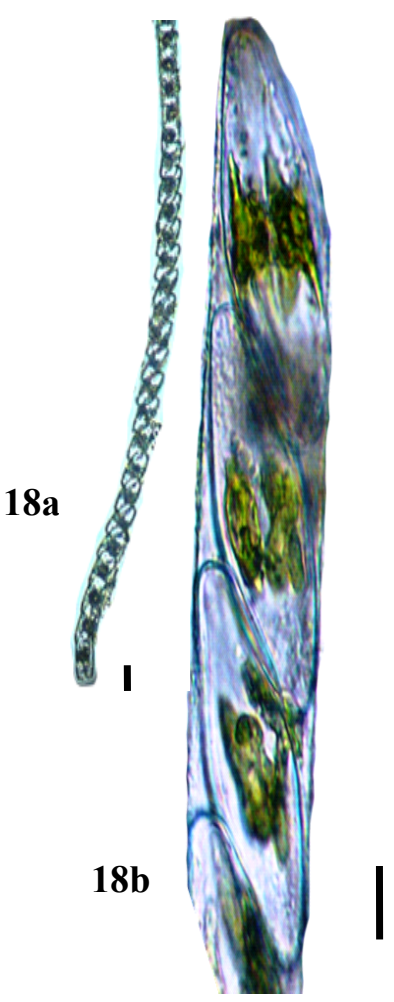

19a

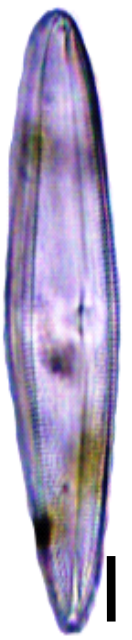

19b
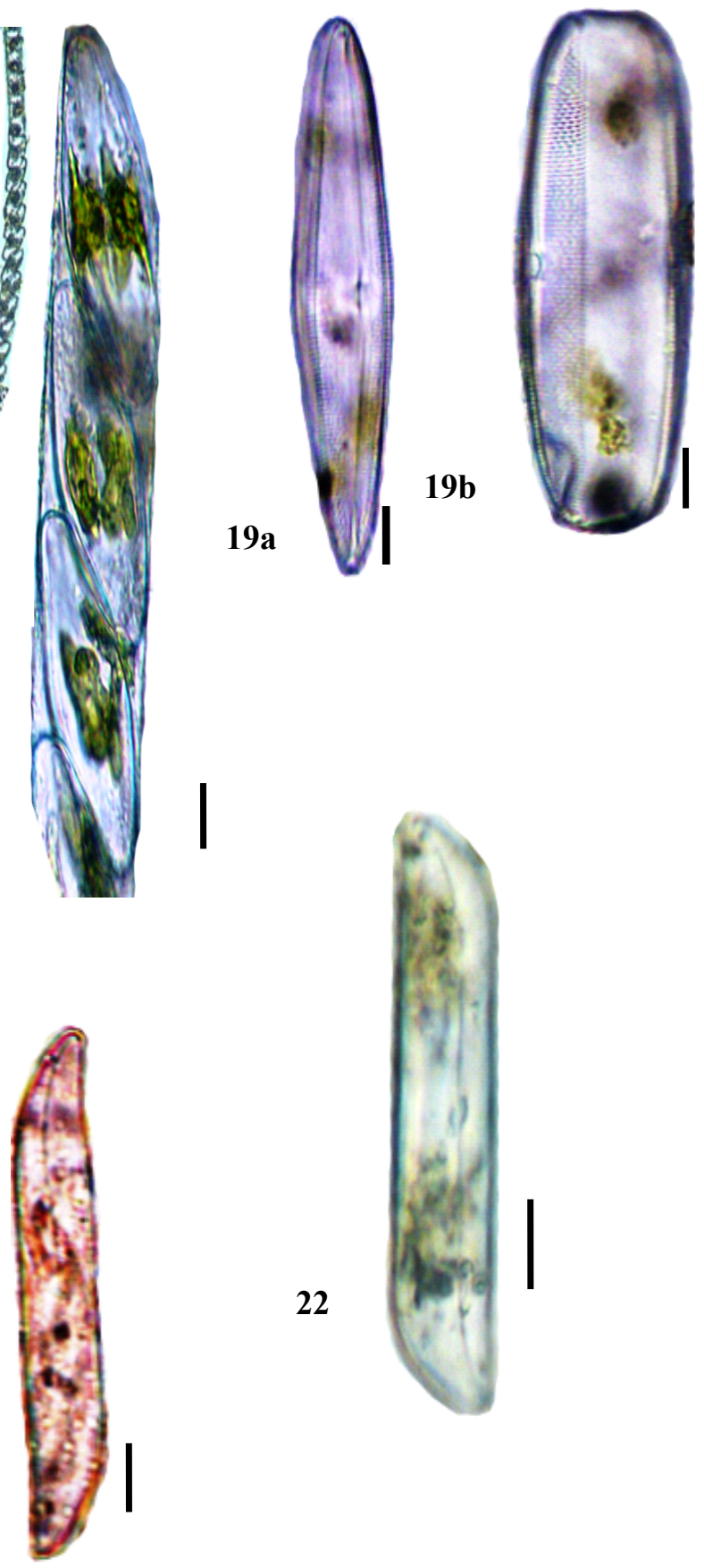

22

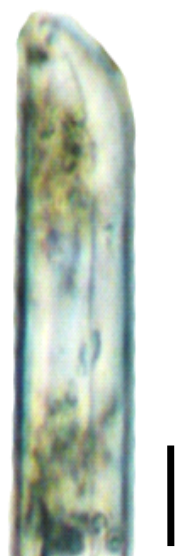

21

Figures 16-22 : 16- Donkinia sp. 17-Navicula humerosa Brébisson, 18a- 18b-Navicula sp., 19a19b-Trachyneis antillarum Cleve, 20-Gyrosigma balticum Ehrenberg, 21-Gyrosigma diminutum (Grunow in Cleve \& Grunow) Cleve, 22-Gyrosigma scalproides Cleve. 


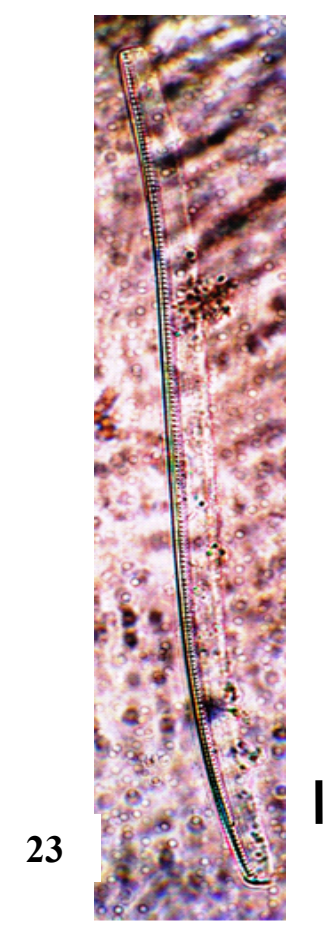

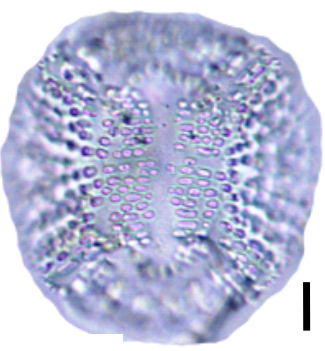

24

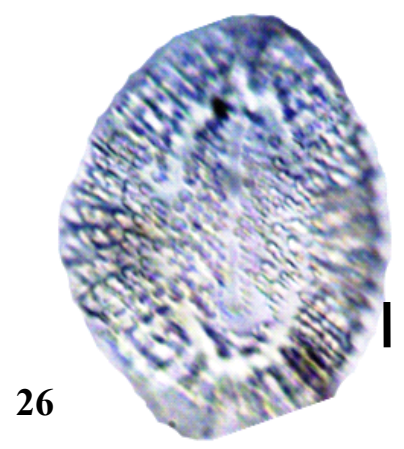

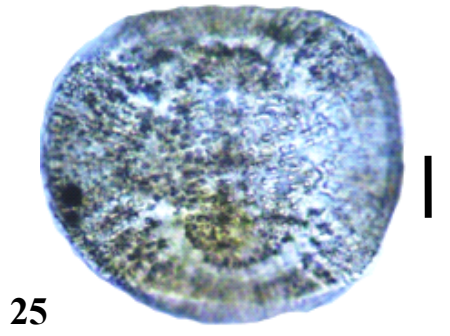

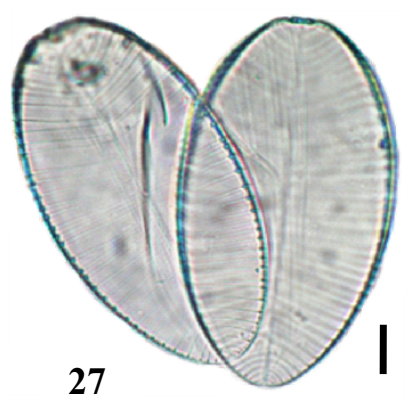

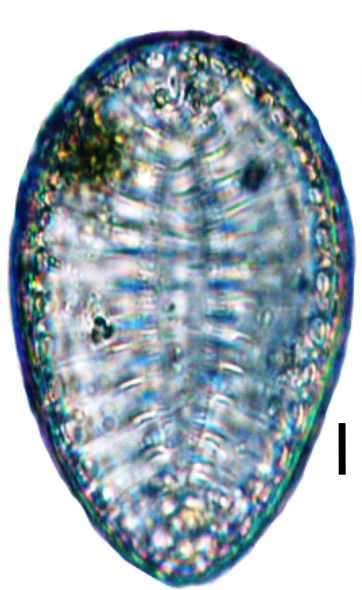

28
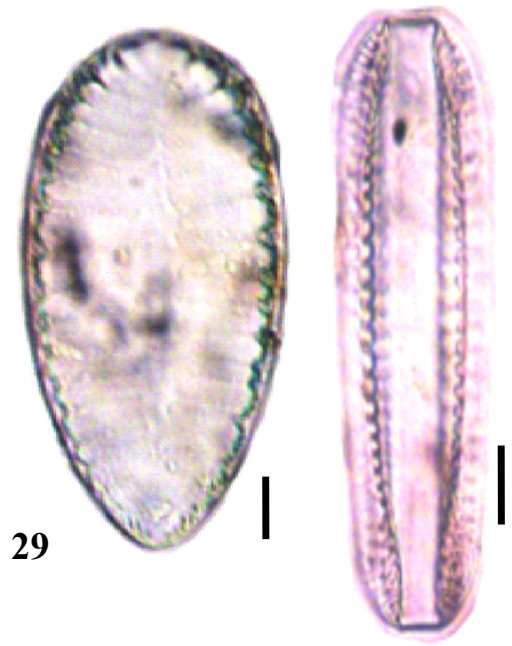

$30 \mathrm{a}$
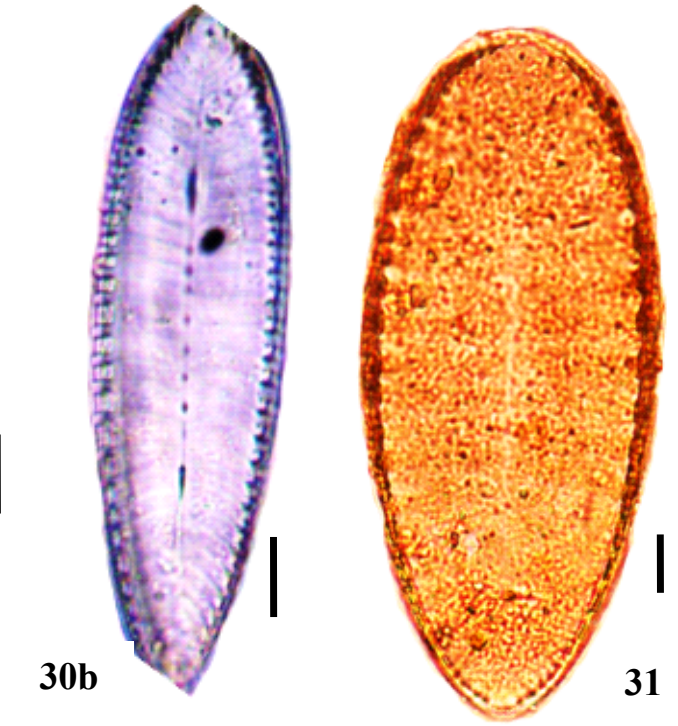

Figures 23-31 : 23-Nitzschia sigma (Kützing) W. Smith, 24-Campylodiscus clypeus Ehrenberg, 25Campylodiscus echeneis Ehrenberg, 26-Campylodiscus eximus Gregory, 27-Petrodictyon gemma (Ehrenberg) Mann, 28-Surirella fastuosa (Ehrenberg) Kützing, 29-Surirella ovata Kützing, 30a-30bSurirella robusta Ehrenberg, 31-Surirella splendida Kützing. 


\section{DISCUSSION}

L'effectif des diatomées pennales de la lagune de Fresco est similaire à celui de SeuAnoï (2012) qui a récolté 29 taxons dans la lagune Ebrié. Cependant, en lagune Aby, 48 diatomées pennales ont été identifiées, et 46 dans la lagune de Grand-Lahou respectivement par Seu-Anoï (2012) et Komoé (2010). Ces différents effectifs pourraient être attribués aux caractéristiques géomorphologiques, à la période (fréquence et durée) d'échantillonnage ainsi qu'à la méthodologie utilisée (filet à plancton plus bouteille hydrologique).

En effet, l'utilisation de la bouteille hydrologique permet de récolter les espèces qui passent à travers les mailles du filet et de retenir les espèces de très petites tailles. Cet instrument n'a pas été utilisé dans notre étude contrairement à celle de Komoé (2010) sur la lagune de Grand Lahou.

Les effectifs les plus élevés ont été observés aux stations 4 et 5 . Concernant la station 4 , située à l'embouchure des rivières Bolo et Niouniourou, cet effectif pourrait être dû au fait que, selon Niamien-Ébrottié (2010), les diatomées sont bien représentées dans les eaux continentales de la Côte d'Ivoire. Les apports de ces eaux continentales dans la lagune de Fresco ont participé à la richesse floristique des diatomées au niveau de cette station. Quant à la station 5 , située près de la mer, selon Issola (2010), la mer exerce une force de succion des eaux continentales entrainant les taxons de ces eaux vers la mer. Cette action pourrait donc contribuer à l'augmentation de l'effectif des taxons au niveau de cette station.

Sur le plan biogéographique, les formes cosmopolites sont nombreuses. Dans la présente étude plus de la moitié des espèces sont cosmopolites et subcosmopolites. En effet, leur aire de distribution s'étend à travers tout ou la plupart des habitats du monde.

Sept taxons sont signalés pour la première fois en Côte d'Ivoire, ce qui constitue une contribution significative à la connaissance de la microflore algale ivoirienne.

\section{Conclusion}

Ces travaux ont permis de connaître les diatomées pennales de la lagune de Fresco, et de renforcer les connaissances de la microflore algale dans les milieux lagunaires de la Côte d'Ivoire. Elle a montré une importante diversité floristique des diatomées pennales de la lagune de Fresco. Trente taxons répartis en 16 genres, 8 familles, 5 ordres et 2 classes ont été identifiés. Parmi ces taxons, 7 sont nouveaux pour la Côte d'Ivoire. Les stations sous influence continentale (station 4) et marine (station 5) ont enregistré les diversités floristiques les plus élevées.

\section{REMERCIEMENTS}

Les auteurs remercient le Centre de Recherches Océanologiques, les universités Félix Houphouët Boigny, Nangui Abrogoua et Lorougnon Guédé pour les travaux de terrain et d'analyses au laboratoire.

\section{CONFLIT D'INTÉRÊTS}

Les auteurs déclarent qu'ils n'ont pas de conflits d'intérêts.

\section{CONTRIBUTIONS DES AUTEURS}

KES : Conception, collecte des données de terrain, rédaction de l'article, ANM : Collecte des données, lecture et correction de l'article. AMP : Lecture et correction de l'article.

\section{RÉFÉRENCES}

Adou ARE. 1999. Contribution à la connaissance des Algues de la lagune Tendo à N'Guiémé (Côte d'Ivoire). Mémoire de DEA, U.F.R. Biosciences, Université de Cocody Abidjan, Côte d'Ivoire, $96 \mathrm{p}$.

Amossé A. 1970. Diatomées marines et saumâtres du Sénégal et de la Côte d'Ivoire. Bull. I.F.A.N. série A, 32 (2): 289-311.

Arfi R, Dufour P, Maurer D. 1981. Phytoplancton et pollution : premières études en baie de Biétri (Côte d'Ivoire). Traitement mathématique des données. Oceanologica Acta, 4: 319-329. 
Bourrelly P. 1961. Algues d'eau douce de la République de Côte d'Ivoire. Bull. I.F.A.N. série A, 23 (2): 283-374.

Bourrelly P. 1975. Quelques Algues d'eau douce de Guinée. Bull. Mus. Nat. d'hist. Nat. $3^{e ̀ m e}$ série Botanique, 20 (276): 171.

Bourrelly P. 1981. Les Algues d'eau douce. In Les Algues Jaunes et Brunes, Chrysophycées, Phéophycées, Xanthophycées et Diatomées (Tome II). Soc. Nouv. Éd. Boubée : Paris ; 517 p.

Campeau S, Dubuc R. 2014. Suivi biologique des cours d'eau de la ville de SaintHyacinthe à l'aide de l'indice IDEC. Indice Diatomées de l'Est du Canada (IDEC, version 3), Rapport déposé à l'Organisme de bassin versant de la rivière Yamaska (OBV Yamaska) et à la ville de Saint-Hyacinthe, $15 \mathrm{p}$.

Carpentier B. 1982. Premières données sur la flore diatomique de la lagune Ébrié (Côte d'Ivoire). D.E.A. d'Algologie, Univ-Paris VI, 15 p.

Chahboune N, Mehdi M, Abidi M, Douira A. 2012. Impact environnemental et évaluation de la qualité des eaux par des méthodes chimiques et biologiques " Diatomées ». Int. J. Biol. Chem. Sci., 6(6): $\quad$ 8024-8033. DOI: http://dx.doi.org/10.4314/ijbcs.v6i6.46

Compère P. 1975b. Algues de la région du lac Tchad. IV- Diatomophycées. Cah. O.R.S.T.O.M., sér. Hydrobiol., 9 (4): 203-290.

Compère P. 1991. Contribution à l'étude des Algues du Sénégal. Algues du lac de Guiers et du Bas-Sénégal. Bull. Jard. Bot. Nat. Belg., 61 (3-4): 171-267.

Couté A, Iltis A. 1985. Étude au microscope électronique à balayage de quelques Algues (Dinophycées et Diatomophycées) de la lagune Ébrié (Côte d'Ivoire). Nova Hedwigia, 41 : 6979.

Dufour P, Pagès J, Lemasson L. 1979. Éléments nutritifs et production primaire dans les lagunes de Côte d'Ivoire. Cycle annuel. Arch. Sci. Centre Rech. Océanogr. Abidjan, 5 : 1-60.

Iltis A. 1984. Biomasses phytoplanctoniques de la lagune Ébrié (Côte d'Ivoire). Rev. Hydrobiol. Trop., 118 : 153-175.

Issola Y. 2010. Étude des caractéristiques climatiques, hydrochimiques et de la pollution en métaux lourds d'une lagune tropicale : la lagune de Fresco (Côted'Ivoire). Thèse de Doctorat unique, Université de Cocody, $177 \mathrm{p}$.

Hans Du Buf, Bayer MM. 2002. Automatic diatom Identification Series in Machine Perception and Artificial Intelligence Vol 51, 316 p.

Komoé K. 2010. Distribution du phytoplancton dans le complexe lagunaire de Grand-Lahou en Côte d'Ivoire. Thèse de Doctorat, Université de Cocody, Abidjan, $282 \mathrm{p}$.

Krammer K, Lange-Bertalot H. 1991. Bacillariophyceae: Centrales, Fragilariaceae, Eunotiaceae, Ettl H, Gerloff J, Heynig H, Mollenhauer D. (eds). Süßwasserflora von Mitteleuropa Gustav Fischer: Stuttgart; 576 p.

Leclercq L, Maquet B. 1987. Deux nouveaux indices chimique et diatomique de la qualité d'eau courante. Application au Samson et à ses affluents (Bassin de la Meuse belge). Comparaison avec d'autres indices chimiques, biogéniques et diatomiques. Document de travail $\mathrm{n}^{\circ} 28$, Institut Royal des Sciences Naturelles de Belgique, $113 \mathrm{p}$.

Maurer D. 1978. Phytoplancton et pollution: Lagune Ébrié (Abidjan) et Secteur de Cortiou (Marseille). Thèse $3^{\text {ème }}$ Cycle, Aix-Marseille II, $121 \mathrm{p}$.

N'Gouran P. 2006. Étude du phytoplancton et des paramètres physico-chimiques de la lagune Ébrié : cas de la baie de Biétry. Côte d'Ivoire, $61 \mathrm{p}$.

Niamien-Ébrottié JE. 2010. Composition et distribution spatiale et saisonnière des peuplements d'algues de quatre rivières du Sud-Est de la Côte d'Ivoire (Soumié, Éholié, Éhania et Noé). Thèse de 
Doctorat, Université d'Abobo-Adjamé, Côte d'Ivoire, $119 \mathrm{p}$.

Peragallo H. 1908. Diatomées de France. Edition Micrographe L-K, $137 \mathrm{p}$.

Plante-Cuny MR. 1977. Pigments photosynthétiques et production primaire $\mathrm{du}$ microphytobenthos d'une lagune tropicale, la lagune Ébrié (Abidjan, Côte d'Ivoire). Cah. O.R.S.T.O.M. Sér. Océanogr., 15 : 3-25.

Reyssac J. 1966. Le phytoplancton entre Abidjan et l'Équateur pendant la saison chaude. Doc. Sci. CRO Abidjan, 2: 1-11.

Round FE, Crawford RM, Mann DG. 1990. The Diatoms: Biology and Morphology of the Genera. Cambridge University Press: New York; 747 p.

Rumeau A, Coste M. 1988. Initiation à la systématique des diatomées d'eau douce pour l'utilisation pratique d'un indice diatomique générique. Bulletin Français de Pêche et de Pisciculture, 309: 1-69.

Saint Martin S, Saint Martin J-P. 2004. Enregistrement par les Diatomées des variations paléoenvironnemantales durant le sarmatien dans l'aire paratéthysienne (Roumanie). Elsevier.

Seu-Anoï NM. 2012. Structuration spatiale et saisonnière des peuplements phytoplanctoniques et variabilité des facteurs abiotiques dans trois complexes lagunaires de Côte-d'Ivoire (Aby, Ébrié et Grand-Lahou). Thèse de doctorat de l'Université Nangui Abrogoua, Côte d'Ivoire, $137 \mathrm{p}$.

Smol JP, Stoermer EF. 2010. The Diatoms: Applications for Environmental and Earth Sciences (2Ed). Cambridge University Press : Cambridge ; p. 667.

Tanoh YV. 2006. Étude du phytoplancton et des paramètres physico-chimiques de la lagune Ébrié : Cas de la baie de Cocody. Côte d'Ivoire, $65 \mathrm{p}$.

Taylor JC, Harding WR, Archibald CGM. 2007. An Illustrated Guide to Some Common Diatom Species from South Africa. Report to the Water Research Commission, South Africa, $225 \mathrm{p}$.

Zongo B, Zongo F, Ouéda A, Couté A, Boussim IJ. 2011. Étude des genres Actinotaenium (Näg.) Teiling et Cosmarium Corda ex Ralfs (Desmidiaceae/Chlorophyta) dans les mares temporaires des régions du Centre, de l'Est et du Nord du Burkina Faso. Int. J. Biol. Chem. Sci., 5(2): 554-569. DOI : http://dx.doi.org/10.4314/ijbcs.v5i2.7211 4. 